\title{
EXPERIENCIA EN METODOLOGÍA CUALITATIVA: HISTORIA DE VIDA
}

\section{EXPERIENCIA IN QUALITATIVE METHODOLOGY: HISTORY OF LIFE}

\section{María del Socorro Borboa-Quintero}

Doctora en Estudios Organizacionales por la Universidad Autónoma Metropolitana, Profesora e Investigadora Titular "C" de la Facultad de Contaduría y administración de la Universidad Autónoma de Sinaloa

\section{RESUMEN}

A partir de la apertura comercial de México las empresas compiten con empresas transnacionales. En el municipio de Culiacán del estado de Sinaloa las pequeñas empresas han sido las más perjudicadas principalmente por su bajo nivel de productividad y competitividad. Por lo que estudiar a dos personas que ha pesar de la complejidad del entorno han influido para el crecimiento y desarrollo de su organización es trascendente para el desarrollo local y estatal. El propósito del presente trabajo, aun cuando se incluyen algunos resultados, es mostrar el método y los instrumentos empleados en la investigación. En primer lugar se plantea el problema a investigar, las preguntas, objetivos e hipótesis que orientan este trabajo, así mismo incluye el diseño de trabajo de campo, que contempla la pertinencia de emplear el método cualitativo de la tradición historia de vida, la recolección y el almacenamiento de los datos, su análisis e interpretación y algunas conclusiones. El estudio permitió determinar las características y cualidades que conforman su personalidad de líder, así como se identifican las prácticas de gestión que han implementado.

Palabras clave: estudio cualitativo, historia de vida, estilo de liderazgo, pequeñas empresas industriales.

Recibido: 04 de diciembre de 2011. Aceptado: 02 de marzo de 2012. Publicado como ARTÍCULO CIENTÍFICO en Ra Ximhai 8(3): 291-309.

\section{SUMMARY}

From the commercial opening of Mexico the companies compete with transnational companies. In the municipality of Culiacan of the state of Sinaloa the small companies have been harmed mainly by his low level of productivity and competitiveness. Reason why to study two people who even with the complexity of the environment they have influenced for the growth and development of its organization which is important for the local and state development. The intention of the present work, even though some results are included, is to show the method and the instruments used in the investigation. In the first place one poses the problem to investigate, the questions, objectives and hypotheses that orient this work, also includes the design of the field work, that contemplates relevance to use the qualitative method of the tradition life history, the collect and the storage of the data, its analysis and interpretation and some conclusions. The study allowed determining the characteristics and qualities that conform their personality of leader, as well as the identification of the management practices that have implemented.

Keywords: qualitative study, history of life, style of leadership, small industrial companies. 


\section{INTRODUCCIÓN}

El futuro de México y en particular el del Estado de Sinaloa depende en gran medida de nuestra capacidad para transformar las pequeñas y medianas empresas en organizaciones competitivas; porque ello permitirá alcanzar un desarrollo económico y social que posibilite elevar la calidad de vida de su población.

Para realizar este estudio se definió el problema de investigación así como los elementos contextuales que lo conforman a partir de una exhaustiva búsqueda a la teoría de la organización, a través de la bibliografía, documentos, algunos servidores web, etc. en la que se refieren las principales investigaciones sobre el área o áreas cercanas, autores, enfoques y métodos e instrumentos empleados tanto para la recolección como para el análisis de la información, conclusiones e interpretaciones teóricas a que llegaron y otros elementos de importancia que permitieron además precisar las principales dimensiones teóricas: 1) organizaciones exitosas y 2) liderazgo que proporcionan sustento a esta investigación.

Posteriormente se procedió a formular las preguntas, los objetivos y las hipótesis de investigación; identificar el método de investigación a utilizar, las técnicas e instrumentos que se emplearían en la recopilación y el manejo de la información, así como a determinar cómo se realizaría el análisis e interpretación de los datos recopilados, indispensables para elaborar el informe de los resultados, como se muestra a continuación:

Planteamiento del problema

La firma del tratado de libre comercio entre Estados Unidos, Canadá y México, la

Son algunos de los problemas detectados a través de aproximadamente 35 diagnósticos realizados por alumnos de la FCA-UAS, quinto grado de la materia administración aplicada, formación de grandes bloques económicos entre países como Japón, Estados Unidos y Europa, y últimamente los tratados de libre comercio de México con Israel, Chile y Europa han provocado un incremento en las importaciones, la inversión extranjera, las alianzas comerciales, etc. Estos hechos han influido para que nuestras empresas compitan internacionalmente con países como Japón y Estados Unidos, los cuales son considerados líderes en el ámbito mundial por sus productos y servicios de calidad y precios competitivos, al utilizar estrategias y técnicas administrativas que les permiten penetrar con éxito en los mercados internacionales.

La formación de bloques económicos y la apertura de los mercados en México ha provocado que muchas pequeñas $\mathrm{y}$ medianas empresas redujeran al mínimo su operación buscando principalmente sobrevivir, y que otras con menos previsión de su futuro desaparecieran; debido a problemas de liquidez, disminución de ventas, cartera vencida, imposibilidad de cubrir créditos bancarios, adeudos fiscales, etc. Problemas diversos que pueden tener origen en las políticas económicas, cambios de gobierno, los embates de la competencia interna y externa, la falta de previsión para enfrentar el futuro (Eroles, 1995). Si bien es cierto que las políticas de gobierno estimulan o desalientan el desarrollo de la pequeña empresa industrial, se considera que es el empresario, particularmente el de la pequeña empresa, es quien requiere de cambios profundos para modificar sus hábitos y replantear sus expectativas y, para lograrlo, los encargados de la dirección y toma de decisiones de las pequeñas empresas requieren contar con ciertas características $\mathrm{y}$ cualidades personales que le permitan en micro y pequeñas empresas del municipio de Culiacán (20002001). 
hacerle frente al cambio y la incertidumbre que ocasiona la globalización.

En México solo el 0.3 por ciento de las empresas se consideran grandes, mientras que el 99.7 por ciento son de tamaño micro, pequeñas y medianas (Rodríguez, 1999).

El Diario Oficial de la Federación, clasifica como pequeña empresa industrial, a aquella que cuenta entre 11 y 50 trabajadores, y señala que las micro, pequeñas y medianas empresas son un elemento fundamental para el desarrollo económico de los países, tanto por su contribución al empleo, como por su aportación al Producto Interno Bruto, representando alrededor del 52\% del Producto Interno Bruto y contribuyendo a generar más del $70 \%$ de los empleos formales.

Desde hace tiempo que se tiene el reconocimiento profundo de que la relación empresarial es el artefacto que maneja la economía de la mayoría de las naciones, principalmente cuando nos referimos al sector primario como la columna vertebral del sistema económico del país; sector que se encarga de agregar valor a las materias primas transformándolas en productos terminados para satisfacer las necesidades de un mercado cada vez más exigente.

A través del tiempo se ha observado que las pequeñas y medianas empresas del municipio de Culiacán, le han proporcionado independencia económica a sus propietarios y la oportunidad de contribuir en el desarrollo económico del país generando empleos, que permiten elevar la calidad de vida de la población mediante los ingresos que perciben los trabajadores.

Información proporcionada por el C.P. Javier Montaño, producto del análisis realizado al trabajo de consultaría y apoyo brindado a la micro, pequeña y mediana empresa en Sinaloa (2000).

Elaborado con información presentada en la ponencia "Los corredores bioceánicos en la integración sub regional y regional de México (Instrumentos para el análisis e implementación de políticas
En el municipio de Culiacán, Sinaloa, México, la pequeña empresa industrial ha sido de las más perjudicadas con la apertura comercial, principalmente por contar con tecnología artesanal o poco automatizada, no contar con suficientes recursos para realizar inversiones importantes en tecnología, falta de preparación de sus propietarios y su resistencia al cambio; escasa o nula previsión del futuro, el esperar resultados a corto plazo, no contar con políticas de reinversión, no estar conscientes de lo que implica la globalización, no asumir riesgos, ser reactivos y no proactivos, etc. Asimismo el director del Centro Regional para la Competitividad Empresarial (CRECE) Sinaloa señala "el empresario-líder es el responsable de todos los problemas que enfrentan las pequeñas empresas, principalmente por su nivel de preparación, el no generar y utilizar información para la toma de decisiones y su resistencia al cambio".

La situación de la pequeña empresa industrial del municipio de Culiacán en el estado de Sinaloa se presenta difícil e incierta y su desarrollo eficiente es una oportunidad de coadyuvar a la expansión del sistema económico del país. Por lo que es prioritario evitar que fracase este tipo de organizaciones, y para ello es importante tomar en cuenta la influencia que tiene el empresario-líder como factor de cambio en la solución de los múltiples problemas que enfrentan. Su gran reto es utilizar con la máxima efectividad y economía los recursos disponibles de la empresa en la elaboración de productos y servicios que la

de integración) presentada por Luis Alberto Hinostroza Fernández y Augusto Gardy Bolívar Espinoza, profesores investigadores de la UAM, Unidad Azcapotzalco, en el 51 Congreso Internacional de Americanistas en Santiago de Chile, julio de 2003. Así como de la página de web del gobierno del Estado de Sinaloa http://www. sinaloa.gob.mx. en consulta realizada en septiembre del 2003. 
gente necesita y desea.

El municipio de Culiacán es la capital del estado de Sinaloa, el cual se localiza al noroeste de México. Su ubicación estratégica le otorga una ventaja para la distribución de sus productos a Estados Unidos con destino a Asia y Centroamérica, así como para la inversión de capital extranjero. Se conecta con los Estados del Sur de EU y Canadá mediante el Área de Libre Comercio de las Américas (ALCA) a través del corredor bioceánico Topolobampo-New YorkVancouver, el cuál tiene como propósito unir el Océano Pacifico con el Atlántico, asimismo la bahía de Topolobampo reúne las condiciones para unir al Océano Pacífico con el Océano Atlántico vía ferrocarril y carreteras. Además desde la ciudad de Culiacán se puede llegar a Nogales, Arizona por carretera de cuatro carriles .

En Sinaloa el $98 \%$ de la industria manufacturera está representado por pequeñas empresas de las cuales el 32.5 por ciento se ubica en Culiacán, dedicadas principalmente a la industria de alimentos, plantas de confección de ropa, compañías embotelladoras e ingenios de azúcar.

También Sinaloa cuenta con ventajas comparativas que lo colocan como líder nacional en el rendimiento de sus cosechas produciendo el 32 por ciento de la producción Agrícola de México. Además posee una de las industrias acuícolas más desarrolladas de México por sus volúmenes de producción, número de granjas en operación, estudios de investigación y personal técnico calificado, actividad que ocupa actualmente sólo el 15 por ciento del área disponible.

Sin embargo y a pesar de los programas de apoyo implementados por la Secretaria de Desarrollo Económico del gobierno del Estado para la empresa micro, pequeña y mediana, falta mucho por hacer para lograr su competitividad.

En este contexto y con el reconocimiento de la problemática que enfrentan, en México la investigación científica de los líderes o propietarios de empresa ha sido escasa o nula.

En el ámbito internacional el líder ha sido objeto de investigación Max Weber en su obra Economía y Sociedad (1922) introdujo el término carisma en su trabajo, señalando que los líderes carismáticos estimulan y transforman a seguidores previamente desanimados, en trabajadores activos elevando su motivación e inspirando un sentido de propósito. Estudios que han servido de base a otras investigaciones, como la realizada recientemente por DeGroot, Timothy Kiker, D Scott Cross, Thomas C. 2000: A meta-analysis to review organizational outcomes related to charismatic Leadership. Evaluaron la relación entre el estilo de dirección carismático y la efectividad de dirección, actuación y satisfacción, esfuerzo, y el compromiso de los subordinados. Sugieren que la dirección carismática produce en los seguidores una internalización fuerte de los valores del líder y las metas de la organización, el compromiso moral a estos valores, y una tendencia de los subordinados a trascender sus propios intereses por causa de la colectividad.

Otro ejemplo se encuentra en el estudio realizado por Munduate, et al., 1999, el cuál se refiere a los modelos de estilos en la dirección del conflicto y efectividad, identificando diferentes modelos para dirigir el conflicto y su efectividad en la aplicación.

Existen otras investigaciones, las cuales sin embargo, no se han orientado a investigar 
a propietarios de pequeñas empresas industriales exitosas; a identificar cuáles son las características y cualidades que conforman su personalidad de líder y las prácticas de su gestión que han influido en el logro del éxito de su empresa en un ambiente de incertidumbre y crisis económica nacional.

Pero sobre todo, no se han enfocado a investigar personas comunes que con base en su trabajo y esfuerzo su empresa a dejado de ser una empresa familiar y se ha convertido en pequeñas empresas que dan trabajo a un número cada vez mayor de personas; el mercado de sus productos, al igual que la infraestructura y equipo que poseen es cada vez más grande, y además lo han hecho en un ambiente de crisis e incertidumbre; de igual manera, no se ha investigado su arribo a posiciones de responsabilidad, la manera en las cuales interpretan y expresan sus tareas a cumplir y el crecimiento personal que puede haber influido en su éxito.

Es clara la necesidad de realizar investigación científica que permita guiar a la organización en forma productiva hacia el futuro.El planteamiento permitió determinar las preguntas, objetivos e hipótesis que guiaron la investigación.

\section{Preguntas de investigación}

1. ¿Cuáles son las características que definen como exitosas a las pequeñas empresas industriales investigadas?

2. ¿Cómo ha sido el proceso de formación personal $\mathrm{y}$ profesional del propietario de la pequeña empresa industrial sinaloense que le ha permitido convertirse en un líder de éxito?

3. ¿Cuáles son las características y cualidades personales que definen el estilo de liderazgo que despliega el propietario de la pequeña empresa industrial exitosa sinaloense?

4. ¿Cuáles han sido las prácticas de gestión que han implementado los propietarios de las pequeñas empresas industriales investigadas que han influido en el logro del éxito de su organización?

\section{Objetivo general}

Conocer el proceso de formación personal y profesional del propietario de la pequeña empresa industrial sinaloense y las características y cualidades que conforman su personalidad de líder, así como, identificar las prácticas de gestión implementadas, para determinar qué ha influido en el logro del éxito de su organización, mediante la construcción de historias de vida a dos empresarios sinaloenses a partir de conocer y analizar lo que hacen, cómo lo hacen y lo que se requiere para hacerlo bien.

\section{Supuesto de investigación}

Elprocesodeformaciónpersonalyprofesional del pequeño empresario industrial sinaloense determina las características y cualidades que conforman la personalidad y capacidad empresarial del líder, indispensables para la implementación de prácticas de gestión que influyen favorablemente en el éxito de su organización.

\section{Método de investigación}

Cuando se tiene definido un problema de interés no solo personal, sino que además éste tiene un impacto social, surge la inquietud de cómo enfocarlo en la búsqueda de las respuestas a nuestros supuestos, intereses y propósitos que nos indican la importancia del objeto de estudio y las posibles maneras de abordarlo. Esto es, elegir un método que nos permita efectuar la investigación. Aquí resultó conveniente hacer las siguientes preguntas ¿qué tipo de método es conveniente emplear en la investigación? ¿El método 
debe ser cuantitativo o cualitativo? Lo que hizo necesario realizar el siguiente análisis.

El método de estudio cuantitativo se recomienda cuando el estudio a realizar es objetivo y sus variables están estructuradas de tal manera que a una muestra al azar se le aplican preguntas cerradas para la recolección de los datos, mismos que se analizarán y cuantificarán a través de herramientas estadísticas; mientras que los métodos cualitativos de investigación son aconsejables en las ciencias sociales cuando el objeto de estudio es subjetivo, es decir, un fenómeno de la vida social, el cuál para su comprensión requerimos recoger datos descriptivos $<<$ las palabras y conductas de las personas sometidas a la investigación $>>$ con el recurso metodológico de observación participativa, entrevistas a profundidad, análisis textual y transcripciones. El término cualitativo sugiere una búsqueda del entendimiento de una realidad mediante un proceso interpretativo que trata de penetrar en el mundo personal de los sujetos, y busca la objetivación en el ámbito de los significados (Taylor y Bogdan, 1987).

Indiscutiblemente, las diferencias entre el enfoque cuantitativo y cualitativo van más allá de la selección de métodos a escoger y están enraizadas en caminos fundamentalmente diferentes de "conocer la realidad" (Mejía y Sandoval, 1999). La investigación cualitativa se dirige a través de un intenso y/o prolongado contacto con un campo o situación de vida. Estas situaciones son típicamente "triviales" o normales, abstraídas de la vida cotidiana de individuos, grupos, sociedades, y organizaciones (Miles y Huberman, 1994).

A pesar de que los enfoques cualitativos y cuantitativos se consideran con frecuencia mutuamente excluyentes, en realidad puede haber mucho traslape. Los datos cualitativos (por ejemplo, respuestas a preguntas abiertas) pueden analizarse cualitativamente buscando los temas comunes o la construcción de modelos de pensamiento acerca de un tema particular, o pueden analizarse cuantitativamente convirtiendo textos (entrevistas, notas de observación, etc.) a códigos numéricos. De la misma manera, datos cuantitativos (por ejemplo, respuestas a encuestas de preguntas cerradas, representadas numéricamente) pueden analizarse estadísticamente o utilizarse con el propósito de interpretar tendencias o correlaciones (Mejía y Sandoval, 1999). No obstante, es necesario señalar que en la práctica algunos investigadores se han percatado de que sus estudios y entendimientos pueden fortalecerse $y$ ampliarse con diseños que combinan ambos métodos.

Lo anterior, permitió determinar que esta investigación es de tipo cualitativo, debido a que las variables a utilizar comprenden las de comportamiento, personalidad y actitud de los líderes; es decir, el lado subjetivo de su vida social a través de una perspectiva sistémica e integral (holística) que busca obtener datos descriptivos de las personas objeto de estudio. Denzin y Lincoln citado por Simon y Cassell (2000), señalan que la investigación cualitativa es un método de multi-enfoque, que involucra un acercamiento interpretativo a su objeto de estudio. Esto significa que investigadores cualitativos estudian las cosas en sus escenas naturales, intentando dar sentido de, o interpretando los fenómenos en términos de los significados que las personas tienen.

Para realizar este estudio en el que se buscó conocer las características del líder de la pequeña empresa industrial exitosa, se determinó que las principales dimensiones 
congruentes con la pregunta principal de esta investigación son las dimensiones de organizaciones exitosas y de liderazgo; las cuales se determinaron mediante el análisis a los enfoques teóricos de contingencia de la teoría de la organización, de las relaciones humanas, y comportamiento humano.

\section{La tradición de historia de vida}

La historia de vida se emplea de acuerdo con Angrosino (1994) cuando un sólo individuo necesita ser estudiado como sugerido por la literatura o cuando ese individuo puede iluminar un problema específico, como el problema de desafiarse intelectualmente. Asimismo cuando el investigador tiene la necesidad de estudiar a este individuo particular, alguien que ilustra un problema, alguien que ha tenido una carrera distinguida, alguien en el reflector nacional, o alguien que vive una vida ordinaria (Creswell, 1998). Como en el caso del pequeño empresario industrial exitoso sinaloense, alguien que ha tenido una carrera distinguida y es triunfador a pesar de los problemas de su entorno.

El propósito en una historia de vida es examinar la trayectoria de una sola persona, a partir de lo que dice él mismo, su familia y las personas que trabajan directamente con él. Esta investigación se enfocó en la historia de vida de dos propietarios de pequeñas empresas industriales exitosas: un empresario que tiene estudios profesionales y otro no; mediante su análisis identificaron las diferencias y lo que tienen en común y les ha permitido ser exitosos. La historia de vida permitió identificar las características y cualidades que conforman la personalidad de los entrevistados, y las prácticas de gestión que han influido en el éxito de sus

La historia de vida es un tipo de entrevista a profundidad en la que el investigador trata de aprehender las experiencias destacadas de la vida de una persona y las definiciones que esa persona aplica a tales experiencias. A través del testimonio oral el investigador obtiene información del ámbito personal, relatos de vida sobre organizaciones a partir del relato detallado de sus acciones y experiencias como sujeto social. En la que él, como sujeto informante, a través del testimonio, el relato, la narración, el recuerdo, la memoria, el olvido, la vivencia etc., parece un constructor de su mundo. Sin embargo, fue necesario estar consciente que algunos de los testimonios aluden a un yo ideal y no necesariamente reflejan una situación verídica (Mejía y Sandoval, 1999), en parte porque los estilos empleadas en la recolección de los datos son clasificados como elementos subjetivos de difícil manejo científico.

Por lo anterior, fue necesario tomar en cuenta lo que señalan Taylor y Bogdan (1987), no aceptar sin sentido crítico la validez de las respuestas otorgadas por los informantes, debido a que es posible que exista discrepancia entre lo que dicen y lo que realmente hacen, y por otro lado; las personas dicen y hacen cosas diferentes en distintas situaciones. Por ello se contrastó la información proporcionada por los empresarios, con la información proporcionada por los subordinados $\mathrm{y}$ algunos familiares que trabajan en la organización.

La tradición cualitativa de historia de vida permitió conocer el proceso de formación personal y profesional de dos propietarios de pequeñas empresas industriales sinaloenses e identificar las características y cualidades que conforman su personalidad de líder que les ha permitido lograr el éxito de su organización a partir de determinar lo que hacen, cómo lo hacen y lo que requieren para hacerlo bien.

sus experiencias e informaciones testimoniales sobre algunos acontecimientos y periodos de tiempo en su vida particular que acontecieron en determinados espacios y contextos histórico socio-culturales, los cuales son siempre matizados por los sentidos y experiencias personales del propio entrevistado (Galindo, 1998). 
Recolección y almacenamiento de los datos De acuerdo con Taylor y Bogdan (1987), en la fase de recolección y almacenamiento de los datos se identificaron tres etapas: la primera relacionada con el establecimiento del rapport, es decir, para lograr el acceso al campo de estudio se necesita establecer relaciones abiertas con los participantes, buscando que se sientan cómodos para que no nos vean como intrusos. La segunda es cuando se establecen estrategias y tácticas de campo que permitan estar en el lugar y momento que se genera la información y la tercera etapa involucra el registro de los datos en forma de notas de campo escritas, tomando en cuenta que no siempre se puede tomar nota o grabar en el momento preciso y que las observaciones son útiles sólo en la medida en que pueden ser recordadas y registradas.

\section{Establecimiento del Rapport}

Plummen (1983) señala que para realizar un estudio biográfico se necesita encontrar un individuo para estudiarlo, un individuo que sea accesible para proporcionar información y distintivo por sus logros y ordinariez o alguien que vierte la luz en un fenómeno específico o del problema que esta siendo explorado y además agrega que el escenario ideal para la investigación es aquel en el cual el observador logra fácil acceso, establece una buena relación con los informantes y recoge datos directamente relacionados con el estudio (Creswell, 1998).

Lograr el acceso y establecer el rapport con las personas que participan como informantes en el trabajo empírico no fue nada fácil, por un lado se tiene que son personas muy ocupadas y por lo regular su tiempo es limitado y por otro son personas que no desean involucrarse porque tienen desconfianza sobre el manejo que se dará a la información; debido a ello no aceptan rápidamente.

Cuando inicié la búsqueda de las personas que formarían parte de esta investigación, me acerqué de manera personal y sin recomendación alguna a pequeñas empresas en las que, a simple vista, se percibe su crecimiento en cuanto a infraestructura, personal y número de clientes. No obstante que en algunas pequeñas empresas se establecieron formalmente fechas para la primera entrevista, ésta no se concretó e incluso algunas veces las secretarias utilizaban diversos pretextos para negar al propietario.

Las personas que forman parte de esta investigación son propietarios de empresas que están en el ramo de la industria de alimentos y se estableció contacto con ellos a través de recomendaciones. El licenciado Elías Gutiérrez propietario de Productos Oasis, S.A. de C.V. fue recomendado por el licenciado Cuahutemoc Celaya, compañero maestro de la Facultad de Contaduría y Administración de la Universidad Autónoma de Sinaloa, quien otorga asesorías a pequeñas empresas de la localidad y por su parte el señor Joaquín Pérez, propietario de Panificadora la Principal fue recomendado por la señora Elvia Elenes Gerente de CANACINTRA.

La primera entrevista fue fundamental para obtener el consentimiento de los propietarios de la pequeña empresa industrial exitosa a participar en este estudio; así como para el establecimiento del rapport, para lo cual favoreció entre otras cosas, el manifestar lo siguiente:

- Darles a conocer el propósito central

En el nombre de la empresa y del propietario se utilizaron pseudónimos, para garantizar el anonimato y la confidencialidad. 
del estudio y los procedimientos que serían usados en la recolección de los datos

- Hacer comentarios que les proporcionaran confianza, como el derecho a proteger su anonimato, y su derecho a retirarse voluntariamente del estudio en cualquier tiempo.

- La promesa de entregarles los resultados del estudio.

Lo anterior fue muy importante para el establecimiento de buenas relaciones, debido a que para la elaboración de las historias de vida se considera necesario realizar estancias en las organizaciones investigadas. Lograr el acceso a la organización fue muy elemental para la recolección de la información de los informantes primarios y secundarios a través de las entrevistas a profundidad y la observación ordinaria-pasiva.

Es importante mencionar que a pesar de los problemas que se enfrentaron para contactar a estos dos empresarios, el esfuerzo realizado se vio recompensado por la elocuencia, amabilidad, disponibilidad y sobre todo, por el interés que mostraron desde el principio para participar en este estudio.

Recolección de los datos

Para la historia de vida, se utilizó como recomienda Creswell (1998), principalmente la entrevista a profundidad y de forma secundaría la observación pasiva y la revisión documental. La observación en esta investigación fue ordinaria-pasiva, es decir se estuvo en el lugar donde se generaron los datos, pero únicamente como espectador, lo que permitió conocer el contexto en el que se desarrollan las personas entrevistadas y comprender mejor su lenguaje.

Por entrevista a profundidad se entiende los reiterados encuentros cara a cara entre el investigador y los informantes, encuentros dirigidos hacia la comprensión de las perspectivas que tienen los informantes respecto de sus vidas, experiencias o situaciones, tal como las expresan con sus propias palabras, por lo que para obtener conocimientos sobre la vida social, los científicos sociales
La información se obtuvo del informante primario, los propietarios de las empresas, $\mathrm{y}$ de los informantes secundarios, los subordinados y los familiares de los empresarios que trabajan en la organización, de tal forma que permitió triangular la información proporcionada por las fuentes. Se tomo en consideración lo que señala Creswell (1998), respecto a que la historia de vida no contempla la totalidad de eventos y facetas que componen la vida de un sujeto, sino que se concibe como el conjunto de información del sujeto a propósito de un evento o eventos que el propio investigador identifica como importantes.

Para asegurar que los temas claves fueran explorados a profundidad se incitó a los entrevistados a extenderse en sus respuestas y teorizar sobre su vida, utilizándose la siguiente guía de entrevistas:

Temas claves utilizados en las entrevistas con los empresarios

1. Relaciones familiares.

2. Escolaridad.

3. Trabajo voluntario.

4. Los amigos.

5. El concepto de sí mismo:

Prioridades.

Distribución de su tiempo.

6. Las metas.

7. Los éxitos/logros.

8. Las tensiones/lfracasos.

9. Empleos:

Puestos.

Función/responsabilidad.

10. El ser empresario:

Responsabilidad con los clientes.

emplean en gran medida los relatos verbales. El instrumento de la investigación no es un formulario de entrevista, sino el propio investigador y el rol que asume implica no sólo obtener respuestas, sino también aprender qué preguntas hacer y cómo hacerlas (Taylor y Bogdan, 1987). 
Responsabilidad con la comunidad.

Análisis de problemas.

Como aprovecha oportunidades.

Relación con los subordinados.

Mecanismos de decisión.

Forma de gestionar.

Estilo de mandar.

Filosofía de la empresa.

Preguntas utilizadas con los subordinados

1. ¿Cuánto tiempo tiene trabajando y en qué puestos?

2. ¿Ha recibido algún tipo de capacitación?

3. ¿Conoce lo que la empresa espera de usted?

4. ¿Conoce las reglas que tiene que cumplir?

5. ¿Cuándo se presentan problemas laborales cómo los resuelve?

6. ¿Ha tenido oportunidad de proponer mejoras para su puesto?

7. ¿Cómo considera el ambiente de trabajo de la empresa?

8. ¿Cómo considera su relación con el propietario de la empresa?

9. ¿Cómo se siente trabajando en esta empresa?

Preguntas utilizadas con los familiares (Esposa e hijos trabajadores)

1. ¿Cómo es su relación con el propietario? Como empleados y como familia.

2. ¿Cuándo existen diferencias cómo las resuelven?

3. ¿Cómo consideras el trato del propietario con los empleados?

4. ¿Algo por lo que consideras se recordará al propietario?

5. ¿El propietario le ha brindado todas las oportunidades, está haciendo lo que quiere?

6. ¿Cuáles son las satisfacciones que le ha brindado esta empresa?

7. ¿Cómo toman las decisiones?

8. ¿Qué hace cuándo no se siente capaz de tomar una decisión, aún cuando sea de su área?

9. ¿Cómo es su relación con los empleados?

10. ¿Qué admiras del propietario?

La guía de la entrevista sirvió solamente para recordar qué preguntas hacer sobre los diversos temas, misma que se revisó y amplió a medida que se avanzó en las entrevistas. Se efectuaron alrededor de 25 visitas a cada pequeña empresa industrial en los periodos comprendidos del 08 de mayo al 13 de junio del 2002 y del 28 de junio al 3 de agosto del 2002, y del 20 de septiembre al 18 de diciembre del 2002, en las cuales se permanecía en la empresa por espacios de tres a cinco horas. Tiempo en el cual se obtuvieron 25 horas de grabación, se realizó registro de las observaciones, de igual manera se examinó información documental relacionada con la organización.

Para registrar la observación pasiva se empleó un diario de campo, y el registro de los sucesos se realizó inmediatamente después de que ocurrieron, para evitar el riesgo de distorsionar la información o que se olvidaran datos valiosos. Para la obtención de la información en las entrevistas a profundidad se utilizó la grabadora, por lo que fue necesario transcribir la información.

Análisis e interpretación de los datos Taylor y Bogdan (1987) señalan que el análisis de datos, implica ciertas etapas diferenciadas. La primera es una fase de descubrimiento en progreso: identificar temas y desarrollar conceptos y propuestas. La segunda fase, propiamente se produce cuando los datos ya han sido recogidos, incluye la codificación de los datos y el 
refinamiento de la comprensión del tema de estudio. En la fase final es cuando el investigador trata de relativizar sus descubrimientos, es decir, de comprender los datos en el contexto en que fueron recogidos.

No obstante que el análisis e interpretación de los datos es una actividad que requiere mayor concentración al final de la investigación, en la historia de vida la recolección y el análisis de los datos son actividades que van de la mano.

Para el manejo de los datos, y para evitar hacer interpretaciones subjetivas, sin evidencia, surgidas de la mera intuición como lo señalan Mejía y Sandoval (1999); se incluyeron todos los datos recabados en las entrevistas a profundidad, revisión documental y la observación pasiva. Este proceso implicó la sistematización de los datos a partir de clasificar, categorizar, organizar y estructurar de manera adecuada y metódica todos los datos recabados, lo cual facilitó hacer el análisis e interpretación más objetivos.

Lo anterior se hizo necesario porque a través de las entrevistas a profundidad, se sigue la pista a temas generales que se consideran útiles a nuestro estudio, mismas que propician respuestas muy amplias de los entrevistados y aunque se tiene el cuidado de centrar las preguntas, en algunos momentos de la entrevista las respuestas se extienden y se tocan otros temas. Por lo que a la par con la recolección de los datos mediante las entrevistas, fue necesario realizar transcripciones, leer y releer las notas de campo, buscando huecos de información, codificando y desarrollando condiciones para comenzar a dar sentido a éstos. La lectura de los datos tuvo el propósito de relacionarlos con las preguntas, objetivos y supuestos de investigación planteados.

Posteriormente se procedió a identificar y separar los datos respecto a las principales etapas, los principales acontecimientos y las principales experiencias de la vida de las personas investigadas. Convirtiéndose así cada etapa o categoría en un capítulo o sección, buscando las relaciones lógicas en las categorías con el propósito de dar coherencia y significado a las historias de vida.

La historia de vida contiene una descripción de los acontecimientos y experiencias importantes de la vida de una persona o alguna parte principal de ella, en las propias palabras del protagonista, de tal manera que mediante el análisis de la información se ha buscado compaginar los relatos en forma coherente a través de la narración, mostrando los sentimientos, modos de ver y perspectivas de la persona. El análisis permitió construir una especie de matriz como lo recomienda Galindo (1998) y Creswell (1998) separando los datos de su experiencia individual, de manera cronológica, lo cual marca los momentos de cambio, de crisis, y de transición de su vida personal; misma que se organizó en ámbitos: origen, familiar, comunitaria, laboral, profesional, empresarial, etc., considerando la totalidad de la información recopilada.

Las categorías encontradas mediante el análisis corresponden al proceso de formación personal y profesional del propietario; el origen y evolución de la empresa; las practicas de su gestión en relación a los procesos de producción y comercialización, los recursos humanos y las estrategias y políticas implementadas; así como la referente a las personalidades de los empresarios, todas ellas consideradas importantes por su influencia en el éxito de 
sus organizaciones, mismas que conformaron la estructura de las historias de vida como se muestra en el esquema siguiente.

Historia de vida

- $\quad$ Proceso de formación personal y profesional

- $\quad$ Origen y evolución de la empresa

- $\quad$ Prácticas de gestión

o Procesos de producción y comercialización

o Recursos humanos

o Estrategias y políticas implementadas

- Misión y visión

- $\quad$ Aprendiendo de los grandes

- Capacitación permanente Conservación de inventarios mínimos

- Diferenciación de los productos y reducción de costos

- Innovación de los procesos

- Investigación de la competencia y participación en el mercado

- Participación en la toma de decisiones

- Determinantes de la ventaja competitiva

- $\quad$ Personalidad del líder

\section{RESULTADOS}

Después de identificar las principales categorías y relaciones lógicas de los datos en ambas historia de vida, se encontró que no todos los datos recogidos se ajustan a las categorías y que incluso en algunas de ellas hubo necesidad de ampliar los datos, mediante entrevistas adicionales. Al respecto Creswell (1998), recomienda para el análisis de datos cualitativos, sobre todo cuando se trata de base de datos especialmente grandes, por ejemplo cuando el trabajo empírico comprende de 20-30 entrevistas extensas como en el caso de las historias de vida, el empleo de programas computacionales como el NUD-IST y el diagrama de árbol, los cuales se han diseñado para satisfacer mayormente las necesidades de un enfoque que de otros; sin embargo, como no se tuvo acceso a ellos, la clasificación y codificación de los datos obtenidos se realizó de manera práctica. Para dar coherencia y claridad a la información obtenida se empleo el estilo narrativo, descriptivo como se muestra en los ejemplos siguientes:

La primera historia de vida se centra en el licenciado Elías Gutiérrez Pelayo propietario de la empresa Productos Oasis, S.A. de C.V. organización que se dedica a la producción y comercialización de bebidas y otros productos comestibles en el municipio de Culiacán, Sinaloa.

Proceso de formación personal y profesional El licenciado Elías Gutiérrez nació el 20 de julio de 1947, en el rancho el Angelín que pertenece al municipio de Villa de Purificación, del estado de Jalisco. Es el quinto hijo de seis hermanos y tenía diez años cuando a insistencia de su madre se trasladaron a Villa de Purificación y pudo iniciar su primaria, no obstante él ya sabia leer y escribir, debido a que su padre se preocupó por llevar maestros de otros lugares para que en su casa les enseñaran a leer y escribir. La relación familiar la recuerda con cariño, menciona que fue una época muy bonita en la que sus padres insistieron en fomentar el compañerismo y el respeto entre los hermanos. Refiere que a pesar de que sus padres no fueron a la escuela y que incluso su padre nunca llego a escribir su nombre, le enseñaron algunas de las siguientes cosas: "A mis padres les aprendí entre otras cosas lo que es el respeto, la importancia del trabajo, el amor al prójimo, así como a tenerle mucho respeto a la naturaleza. Mi padre era un hombre de campo; pero un hombre que entendía el campo, él cortaba madera y 
sabia cuándo cortarla y por qué cortarla, no tumbaba un árbol nomás por cortarlo".

"Mi madre, un tanto contraria al campo, ella era más comerciante; pero siempre hacía las cosas bien; ella decía que cuando se saca ventaja de un negocio se debe reinvertir y que cuando eres parejo, cuando eres justo, te abunda. También me decía, puedes recibir más de lo que das, que de lo que haces".

El licenciado Gutiérrez comenta que le gusta el campo, el ganado, puesto que ahí nació, pero que después de largas jornadas de trabajo, llegando enlodado y mojado por el sudor o la lluvia a su casa, sentía que eso no era lo que el quería para su futuro, además, veía que en la ciudad las personas andaban mejor vestidas, trabajando en la sombra, y comiendo a sus horas. Por lo que, cuando su madre insiste para que continúe estudiando, deja su pueblo y se traslada a Autlán, Jalisco, con el propósito de estudiar y ser autosuficiente, porque no contaba con el apoyo de su padre. Es ahí donde se emplea en un restaurante trabajando de mesero y cocinero para pagar sus estudios; después, cuando termina sus estudios en la escuela de comercio, trabaja primero en un banco y posteriormente en Teléfonos de México, de su experiencia en Bancomer comenta:

"En Bancomer, entré primeramente de cajero ambulante, y de ahí me pasaron a ayudante de cobranzas. Cuando salió de vacaciones el encargado de cobranzas, me quedé en su lugar y tuve mucho contacto con la secretaria de la gerencia porque estábamos muy juntos. Además, como tenía más velocidad que ella en la máquina de escribir, con mucha frecuencia le ayudaba o checaba la redacción. Cuando terminan las vacaciones del encargado de cobranza, ella me propone para que la supla cuando le dan vacaciones. El gerente aceptó, y desde ahí estuve en todos los departamentos, eso me ayudó a tener una visión muy amplia de lo que era el banco. Al presentarse esta situación, me empezaron a jalar de otras sucursales"....

Origen y evolución de la empresa

La Empresa Productos Oasis, S.A. de C.V. nace como empresa familiar en Culiacán, Sinaloa en agosto de 1988, el licenciado Gutiérrez comenta que:

"La idea de tener un negocio propio surge cuando mis hijos empezaron a crecer y no tenía tiempo de atenderlos, debido a que el puesto que tenía en Teléfonos de México absorbía demasiado tiempo, algunas veces salía de mi casa a las siete de la mañana y regresaba a las doce de la noche, incluso los domingos tenía guardias escalonadas, además de que con frecuencia salíamos de una ciudad y nos íbamos a otra y a otra. Entonces supe, por un lado que no estaba con ellos y por otro, me preguntaba ¿qué les voy a enseñar?"

"Cuando renuncié a Teléfonos de México, tenía buen puesto, era Gerente Comercial de Área, contaba con 17 años de antigüedad y muy buena trayectoria, Sin embargo, ya tenía unos tres o cuatro años muy inquieto, porque veía que después de la secundaria de mis hijos, y sobre todo en la carrera ya no podría moverme con facilidad".

El licenciado Gutiérrez explica, quien tiene cierta experiencia en negocios, puede pensarlo más, enfocarlo más, antes de instalar una empresa; pero en su caso él buscaba estar con la familia y enseñarles a trabajar, más que el crecer como empresa.

En el crecimiento de la empresa, el licenciado Gutiérrez considera que su esposa e hijos han tenido una contribución muy importante. Su esposa es la señora Delfina Chávez y están casados desde 1971, tienen tres hijos, Alejandra quien es licenciada en 
Finanzas; Luz Edith, licenciada en Ciencias de la Comunicación, quien está casada y vive en Guadalajara y Cesar Elías quien es odontólogo. Comenta que con su esposa e hijos mantiene una relación basada en el respeto y la confianza y que...

\section{Prácticas de gestión}

Los procesos de producción y comercialización

El licenciado Gutiérrez expresa que iniciaron la producción de una manera muy sencilla, haciendo el producto como lo hacia el señor que le vendió la formula en El Grullo, Jalisco. Menciona que el señor utilizaba envases y tapas de desecho, y cuando la Secretaria de Salubridad lo prohibió, utilizaron bolsas amarradas. Él tenía la idea de utilizar bolsas rotuladas, pero selladas como los "shampoocitos", por lo que, en la ciudad de Guadalajara, buscó un proveedor que cumpliera sus especificaciones de diseño. Sobre el equipo que conforma la planta, comenta:

"Todo el equipo que usted ve aquí, (el que se utiliza para la elaboración y envasado de los productos), se hizo en la localidad de acuerdo a especificaciones. Lo único que mandamos hacer fueron tinacos, tarjas; pero, lo que son las instalaciones incluso de los filtros las hice yo".

También señala que gran porcentaje del equipo que actualmente se emplea en la empresa es diseñado por él y fabricado por un herrero de la localidad de acuerdo a sus especificacíones. Explica que él ha asistido a exposiciones de maquinaria y equipo, pero que éste es muy costoso; por lo que se trae la idea, y la adecua a los materiales de acuerdo a sus necesidades. Además explica que...

\section{Recursos humanos}

En este momento la empresa Productos Oasis, S.A., cuenta con 33 empleados, además de las cinco personas de la familia; pero comenta que en temporada de frío, el número disminuye a quince $\mathrm{y}$ respecto a su personal el entrevistado explica que a la empresa llega gente con diferentes costumbres, y que definitivamente entre más crece el número de empleados en la empresa es más difícil mantener un buen ambiente de trabajo.

"Tal vez porque a la empresa le conviene, buscamos influir e inculcar los valores del trabajo, respeto, y disciplina a nuestro personal. Se pretende que la gente se acostumbre a lo que aquí se hace, por ejemplo, nosotros trabajamos con una capacitación que nos dio el Centro de Ciencias de Sinaloa que se llama $5 \mathrm{~S}$, en la que nos hablan de orden, limpieza, etc., de tal manera que cada empleado tiene la obligación de mantener su área limpia, y la mejor manera de mantener limpia un área limpia, es no ensuciándola. Llega un momento en el que se empieza a respetar al grado de que consciente no lo hace, lo hará inconsciente o lo hará de mala gana, con toda intención; pero, no porque no sabe o no entiende"....

Estrategias y políticas implementadas Misión y visión de Productos Oasis, S.A. de C.V.

A raíz de un curso que les otorgó el Centro de Ciencias de Sinaloa, se elaboró la misión y la visión de la empresa, textos de éstas se encuentran colocados en la pared de la sala donde se reúnen regularmente para análisis de problemas o para otorgar capacitación, explicando el licenciado Gutiérrez que fueron elaborados por ellos mismos y que se hace lo posible por que los empleados los interioricen. Misión:

Somos una empresa interesada en ofrecer productos alimenticios de la más alta calidad 
para satisfacer las necesidades de nuestros clientes. Que busca de manera continua: el beneficio de todos sus integrantes, ser aceptados por los consumidores, la mejora de sus procesos y el desarrollo de la entidad. ...

\section{Aprendiendo de los grandes}

El licenciado Gutiérrez siempre ha mostrado interés por conocer lo que hacen otras empresas, principalmente las grandes y ha tenido la oportunidad de visitar empresas como Coca-Cola, MASECA y JAPAC, comenta que son empresas de las cuales se puede aprender mucho. Por ejemplo:

"Coca-Cola maneja un producto similar al mío; pero por ejemplo en MASECA me quedé sorprendido de la calidad del proceso, y me di cuenta de que es totalmente falsa la idea de que usan también el olote; en JAPAC tienen dos factores que chocan totalmente, una es el área política, las cabezas, y otra el área sindical, debido a que estos últimos tienen muchos vicios, más que un espíritu de progreso. Y si a pesar de ello, han logrado introducir cambios para mejorar el servicio al cliente, no cabe duda que tienen doble mérito"...

\section{Capacitación permanente}

El propietario de Productos Oasis manifiesta que siempre ha considerado que la preparación personal es muy importante para alcanzar las metas que se plantea, por lo que a lo largo de su vida ha aprovechado todas las oportunidades de capacitación que se le han presentado.

Explica que para hacerle frente a los retos que le representa ser empresario, a tomado diferentes cursos, uno de ellos, el de desarrollo empresarial fue dirigido a micros y pequeñas empresas y fue promovido por el Gobierno del estado y la Secretaria de Desarrollo Económico.
"Ese curso de desarrollo empresarial nos habló de que ubiquemos bien cuál es el papel que te gusta realmente, te gusta hacerla de dueño, te gusta hacerla de vendedor, te gusta hacerla de administrador de la empresa, te gusta hacerla de operario, de qué te gusta para que tú te dediques a eso y de ahí en adelante contacta a la gente. Qué le hace que te caiga mal, pero que sepa lo que está haciendo.

Para la capacitación del personal, la empresa cuenta con una sala en la que algunas veces, regularmente los sábados de 7:00 a 8:30 hrs., se otorga capacitación a través de videos, conferencias o talleres impartidos por instituciones como el Centro de Ciencias, particulares, CANACINTRA, CANACO, CIMO, el Centro Empresarial, o por ellos mismos, utilizando rotafolio, proyector de acetatos, películas, etc. Es un lugar agradable y cómodo que está en la parte alta del edificio con vista a la calle, en él se tienen aproximadamente treinta sillas, un escritorio, un rotafolio, y un pintarrón, comenta:

"Aquí traemos desde un sacerdote, un agente de tránsito, un policía, un químico, o alguien que hable de procesos, control de calidad o de relaciones humanas. La variedad de los invitados, es porque todos nos sirven...

\section{Personalidad del líder}

En esta sección de la historia de vida, se identifican las características que conforman la personalidad del licenciado Elías Gutiérrez Pelayo que han influido para lograr el éxito de su empresa "Productos Oasis S.A. de C.V.". Es necesario comentar que a partir de los años treinta se han realizado numerosas investigaciones con este mismo objetivo; no obstante, los descubrimientos acumulados han llevado a concluir que poseer algunas de estas características incrementan la 
probabilidad de triunfar como líder, pero que ninguna de éstas garantiza el éxito. Además, es ineludible precisar que dichos estudios se han llevado a cabo en un contexto económico, político, social y cultural diferente al nuestro, es por ello la importancia de esta investigación.

Una de las características que distinguen al propietario de Productos Oasis es su tenacidad, la cual se ha visto reflejada en todas las acciones que realiza para alcanzar sus metas empresariales, familiares $\mathrm{y}$ personales. Advertimos que su perseverancia lo ha llevado a superar una serie de obstáculos para poder obtener un titulo profesional, comenzando con separarse de la familia cuando todavía era muy joven, trasladándose a un lugar que le brindara esa oportunidad, así como el tener que trabajar para pagarse sus estudios. Motivado por el director de la escuela primaria y con el apoyo de su madre inicia esta aventura a los 16 años y comenta que cuando termina los estudios de comercio, y ya trabajando en un banco "se me atravesó el destino", una esposa y posteriormente tres hijos, por lo que su meta de obtener un título universitario se alejaba más y más. Sin embargo, su tesón le posibilita obtener dicho título a los cuarenta años de edad, en el año de 1987. Al respecto su hija Nora comenta:

"Lo que más admiro de mi papá es su humildad y su tenacidad. Su humildad porque él puede convivir perfectamente desde con los empleados de la planta hasta con el gobernador y a los dos los trata de la misma manera, les da su lugar por ser personas, no por el rango que tengan, y por su tenacidad porque a pesar de que hay altibajos en la vida, cuando se le han presentado obstáculos nunca se da por vencido, él siempre está buscando qué hacer para salir al mercado, qué nuevos productos ofrecer y a pesar de que sus estudios son en Administración
Pública y nunca haber estudiado ingeniería siempre esta perfeccionado lo que se hace en la planta., pues a iniciativa de él muchos de los procesos se han semi-automatizado. Asimismo, siempre se está preparando, y el estarse preparando constantemente, le hace estar actualizado y no irse quedando en el camino".

La primera etapa de trabajo con la información obtenida fue transcribir las entrevistas tal como se obtuvola información, la segunda fue dar coherencia y claridad a ambas historias de vida, de acuerdo a las categorías identificadas, como se observa en los ejemplos anteriores. Pero como la investigación no se agota en el registro de la realidad. Los datos no "hablan", se les hace hablar, fue necesario interpretarla. La interpretación es el resultado del diálogo entre los supuestos teóricos-metodológicos del investigador con la realidad observada. El modo en que uno interpreta sus datos depende de los supuestos teóricos que el investigador ha asumido durante la etapa de análisis intensivo de la investigación, lo cual lleva a buscar perspectivas, significados $\mathrm{y}$ definiciones sociales. El análisis y tratamiento de las entrevistas se realizó desde las perspectivas EMIC y ETIC. La primera identifica la manera como el mundo es concebido, sentido y construido por los entrevistados dentro de un tiempo y espacio concreto; mientras que la versión ETIC es la manera como ese mundo es percibido, sentido y construido por el observador o entrevistador, los cuales se influyen e interrelacionan en la construcción de un cuerpo de conocimiento que da respuesta a diversos problemas sociales.

Como en esta investigación se incluyen dos historias de vida primero se realizó el análisis e interpretación vertical de las categorías que conforman cada una de ellas con relación al 
marco teórico, lo cual obligó a regresar al marco teórico para incluir aquellas teorías que originalmente no se contemplaron y que eran indispensables para realizar el análisis. Posteriormente el análisis se efectuó de manera transversal; esto es, analizar una a una las categorías entre las dos historias de vida con el propósito de compararlas y presentar los resultados de la investigación, como se muestra enseguida.

\section{ANÁLISIS DE RESULTADOS}

En este capítulo se realizó un análisis comparativo a las historias de vida de los propietarios de las pequeñas empresas industriales investigadas, quienes como líderes y responsables de las funciones de dirección de sus organizaciones han influido en su éxito. Tomando en consideración las siguientes categorías: 1) proceso de formación personal y profesional del propietario, 2) origen y evolución de su empresa, 3) prácticas de gestión implementadas y 4) personalidad del líder. A continuación a manera de ejemplo se contempla cómo se trabajo la primera categoría.

Proceso de formación personal y profesional Los padres de ambos propietarios fueron personas humildes con escasa o nula educación, pero quienes se preocuparon por promover un ambiente familiar de hermandad, apoyo y trabajo, orientado por principios muy rígidos en el cual se les inculcó entre otros valores la honestidad, la responsabilidad, el respeto y su fe en Dios. Asimismo, se observa cómo intervinieron desde pequeños uno y otro apoyando la realización de las actividades necesarias para proveer lo indispensable en el hogar.

También se advierte que a pesar de realizar un trabajo que exigía un gran esfuerzo físico y de las largas jornadas empleadas en su ejecución crecieron con muchas necesidades económicas, lo cual permitió que ellos se compararan con familias o personas que poseían una mejor calidad de vida, las cuales tenían la oportunidad de vestir de forma elegante y comer a sus horas, permitiéndoles visualizar y anhelar un futuro mejor, diferente a lo vivido, y para lograrlo pusieron todo su compromiso, dedicación y esfuerzo no obstante las dificultades que se les presentaron en su trayecto al éxito.

Los propietarios de las empresas investigadas obtuvieron su negocio de muy diferente forma.

Por un lado se advierte que el licenciado Elías Gutiérrez establece su organización en el año de 1988, cuando tenía 41 años de edad, una larga experiencia como subordinado en dos empresas nacionales y con un recién logrado título profesional. En su experiencia como subordinado ocupó diversos puestos que le dieron la oportunidad de enfrentar diversas situaciones y aprender de ellas. Para establecer su empresa Productos Oasis, S.A de C.V., su propietario utilizó los bienes que poseía en ese momento para invertir y hacer realidad un sueño que imaginó durante largo tiempo.

Por otro lado, el señor Joaquín propietario de Pastelería la Principal, S.A. de C.V., tomó posesión de una micro empresa familiar en el año de 1952 fundada por su abuelo en el año de 1898, cuando tenía solamente 21 años de edad y de alguna manera presionado por su padre. Empresa que a pesar de contar con una tradición de 54 años en la que habían participado dos generaciones, $\mathrm{su}$ proceso productivo era totalmente artesanal y además, se encontraba en una mala situación económica. En ese momento él contaba con un diploma que acreditaba sus estudios de comercio y con poca 
experiencia como subordinado en las áreas contable y administrativa de una compañía constructora.

El licenciado Gutiérrez tuvo un largo periodo de aprendizaje como subordinado, pero, como lo señalan Kast y Rosenzweig (1988), contar con una experiencia como seguidor durante veinte años o más, no es la mejor forma de preparar a una persona para la responsabilidad de liderazgo. Sin embargo, consideran que el rotar a través de diferentes puestos y relaciones en una organización ayuda a preparar al personal para el rol de liderazgo, debido a que esta situación le permite a la persona experimentar diversas relaciones superior-subordinado y evaluar su efectividad en diferentes situaciones, en este sentido tenemos que ambos propietarios tuvieron la oportunidad de ocupar diferentes puestos, el licenciado Gutiérrez en las dos grandes empresas en las que trabajó y el señor Pérez en la empresa propiedad de su padre.

Aunque el señor Guzmán admite que su relación como subordinado se presentó en la etapa cuando prestó sus servicios en la constructora, el apoyo brindado a la empresa propiedad de su padre, se considera, facilitó la oportunidad de que mantuviera una relación de superior-subordinado lo cual le permitió tener contacto con los clientes, los proveedores, el proceso productivo, etc., y como señalan Davis y Newstrom (2001) para relacionarse eficazmente tanto en dirección ascendente como descendente los líderes deben ser capaces de vestir ambos uniformes (superior-subordinado), además de considerar que la capacidad para seguir a otros es un requisito indispensable para ser buen líder. En el periodo en el cual el señor Benjamín apoyó el trabajo de la panadería, contó con instrucción directa y explicita por parte de su padre, como señalan, Kast y Rosenzweig (1988), la instrucción explícita puede ser útil en el proceso de desarrollo de los líderes.

\section{CONCLUSIONES}

Lo anterior, permitió elaborar las conclusiones del estudio, para lo cual se considero importante establecer apartados temáticos que permitiera explicar si las preguntas de investigación se respondieron, los objetivos se alcanzaron y si las hipótesis se verificaron o no.

La importancia de investigar a propietarios de pequeñas empresasindustriales del municipio de Culiacán, que aún en ambientes de turbulencias y crisis económicas provocadas por la mundialización, sus organizaciones son exitosas, radica en primer lugar, en la originalidad del estudio porque no existen antecedentes de investigaciones dirigidas al líder de las PyMES locales; en segundo lugar, porque las historias de vida obtenidas han permitido identificar características $\mathrm{y}$ cualidades que conforman su personalidad de líder, así como las practicas de gestión que han influido en el éxito de sus pequeñas organizaciones, mismas que orientarán las acciones de otros pequeños propietarios que deseen el éxito de sus empresas.

"El proceso de formación personal y profesional de los propietarios de las pequeñas empresas industriales sinaloenses investigadas ha influido favorablemente en el éxito de su organización”, encontrándose además, la gran influencia que ha tenido en el éxito de sus organizaciones la experiencia de ambos empresarios como subordinados y su gran impulso personal para hacer realidad su sueño.

El éxito de la pequeña empresa industrial sinaloense depende en gran medida de la visión y el impulso personal de los 
propietarios de la misma, quienes a través de la acción del liderazgo involucran a un grupo de personas cada vez más numeroso, hacia el logro de los objetivos de su organización.

La investigación permitió alcanzar el objetivo específico número tres "conocer las características y cualidades que conforman la personalidad de los pequeños empresarios exitosos investigados e identificar el estilo de liderazgo que emplean para determinar si éste ha influido en el éxito de sus organizaciones" se identificaron características personales comunes en ambos empresarios que han influido en el éxito de sus organizaciones. No se puede asegurar si los poseen desde su nacimiento o los han adquirido y perfeccionado con el tiempo. Sin embargo, se observa que el licenciado Gutiérrez y el señor Pérez poseen características señaladas como comunes en investigaciones anteriores, por ejemplo si bien es cierto que desde chicos ellos ambicionaban una mejor calidad de vida, con más comodidades; si no hubiesen tenido un alto impulso personal no lo habrían logrado. Además, ambos propietarios son personas íntegras que crecieron en familias con principios muy rígidos; que les inculcaron entre otros, los valores del trabajo, honestidad, justicia y respeto, los cuales se manifiestan en sus relaciones interpersonales con los subordinados, clientes, proveedores, gobierno y comunidad; mismos que son congruentes con algunos de los rasgos identificados en investigaciones recientes como son un alto nivel de impulso personal, el deseo de dirigir, la integridad personal, capacidad cognoscitiva, creatividad y originalidad, carisma, honestidad y la seguridad en uno mismo, señaladas por Robbins (1999).

Los rasgos y comportamientos de los líderes definen un estilo de liderazgo que no se explica con un sólo enfoque de la teoría existente. Detectándose que éste es un híbrido de los varios enfoques propuestos por la teoría del liderazgo y da origen a un nuevo modelo que podría denominarse innovador o emprendedor, debido a características personales de las personas investigadas.

La figura 1 muestra el modelo de liderazgo que incluye el comportamiento de los líderes investigados con sus subordinados y una propuesta que les permita promover el desarrollo de su pequeña empresa.

Por un lado el estilo de liderazgo que emplea el licenciado Gutiérrez se considera centrado en los subordinados y corresponde con el continuo del comportamiento propuesto por Kast/Rosenzweig (2000). Asimismo se relaciona con las cuatro opciones de liderazgo del modelo ruta-meta propuesto por Robert House y citado por Davis/Newstrom (2001). Por otro lado se observa que el señor Pérez utiliza el estilo de liderazgo centrado en el jefe, el cual se contempla en el lado inverso del estilo empleado por el licenciado Gutiérrez del continuo de liderazgo propuesto por Kast/ Rosenzweig (2000). Mientras que el modelo ruta-meta propuesto por Robert House y citado por Davis/Newstrom (2001) se relaciona con tres de las cuatro opciones que ofrece para ejecutar la acción del liderazgo.

Además considero que aun cuando en las prácticas de ambos propietarios se contemplan elementos de los estilos de liderazgo transaccional y transformacional, su estilo no corresponde a ninguno de ellos.

Aun cuando el estilo de liderazgo del señor Guzmán es centralizado, ha funcionado debido al apoyo que brinda y los incentivos que otorga a sus subordinados, se considera 


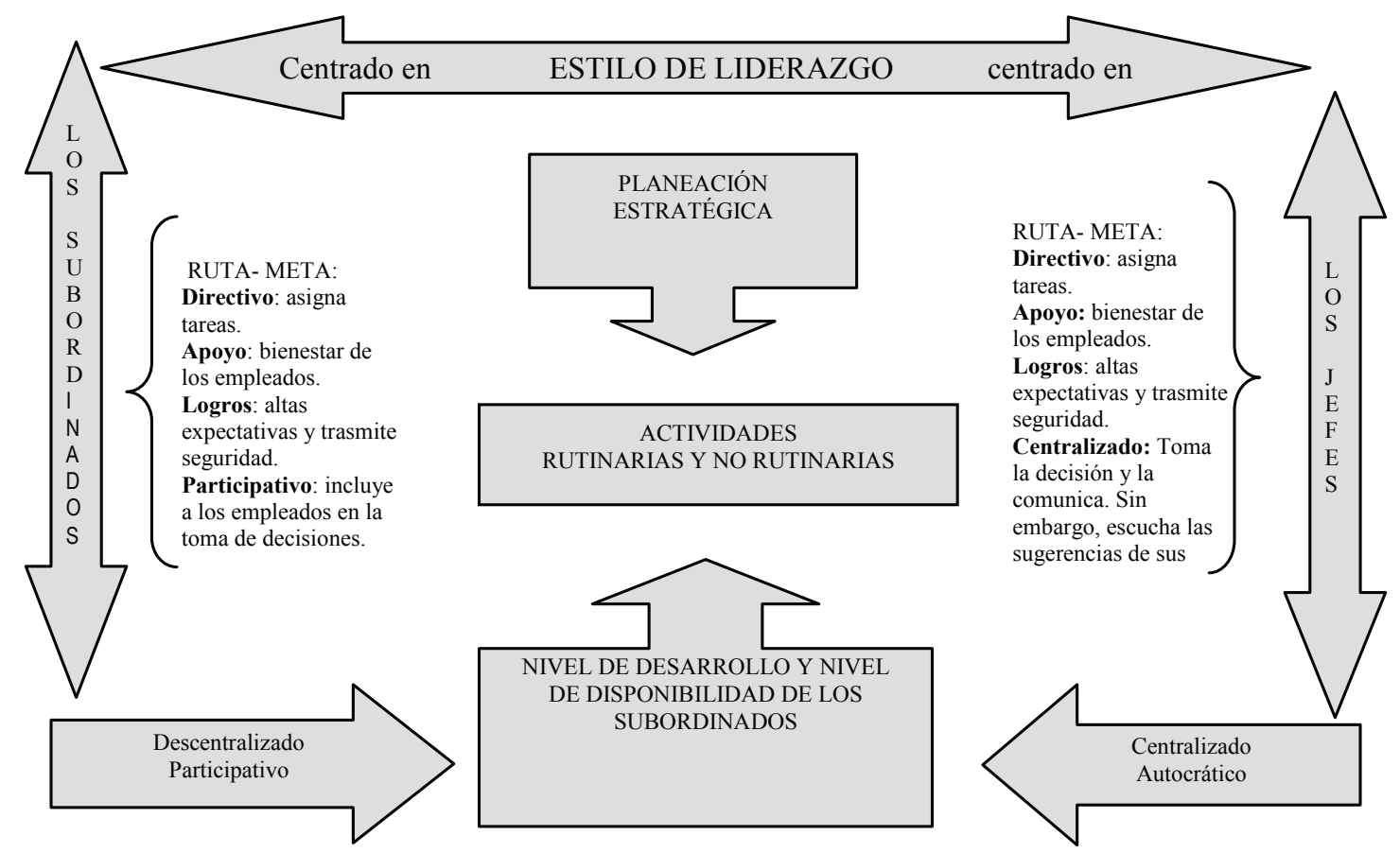

Figura 1. Propuesta de modelo de liderazgo para el desarrollo de la pequeña empresa industrial.

al igual que Paul Hersey y Kenneth Blanchard, citado por Robbins (1999) y Davis/Newstrom (2001) que debe aprender a ajustar su comportamiento en situaciones especificas en la medida en que sus subordinados se vuelvan más capaces y dispuestos a realizar la tarea.

$\mathrm{Si}$ ambos empresarios capacitan constantemente a sus subordinados, por qué no confiar en que puedan decidir lo concerniente a su puesto o con los puestos que se relacionan. Esto es importante, porque si quieren continuar con el crecimiento y desarrollo de sus pequeñas empresas industriales, es necesario que deleguen autoridad de acuerdo al nivel de desarrollo y disponibilidad de sus trabajadores, pueden empezar con las tareas rutinarias y poco a poco ceder en las no rutinarias, en la medida que descentralicen la autoridad podrán ocuparse de la elaboración de planes estratégicos.
Con sus resultados no se intenta generalizar un determinado perfil para todos aquellos pequeños empresarios que deseen tener éxito en su organización; su propósito es proveer al lector de información sobre la formación personal y profesional, así como las prácticas de gestión empleadas, el estilo de liderazgo, y las características personales de dos hombres en los desafíos diarios de su experiencia como líderes de pequeñas empresas industriales.

\section{LITERATURA CITADA}

Aken, Joan. 2000. Qualitative Methods and Analysis in Organizational Research: A practical Guide. (Review). Consultado en 12/15/2000 en http:// www.findarticles.com

Barba Álvarez, Antonio. 2001. Calidad y Cambio Organizacional: Ambigüedad, Fragmentación e Identidad. El caso del laboratorio 
de Pruebas de Equipos y Materiales, Tesis inédita presentada para obtener el grado de Doctor en Estudios Organizacionales. México: Universidad Autónoma Metropolitana.

Carless, Sally A. 1998. Gender differences in transformational leadership: an examination of superior, leader, a subordinate perspective. Summarize of 11 paginate. Consultado el $02 / 15 / 2000$ en http://www. findarticles.com

Creswell, J. W. 1998. Qualitative inquiry and research design: Choosing among five traditions. Thousand Oaks, CA: SAGE Publications, Inc.

DeGroot, Timothy, D. Scott Kiker et Thomas C. Cross. A Meta-analysis to Review organizational outcomes related to charismatic leadership, Canadian Journal of Administrative Sciences, Revue canadienne des sciences de l'administration v17, n4 (décembre 2000), p. 356-371.

Eroles, G. Antonio. 1995. La Pequeña empresa exportadora. México: Panorama Editorial.

Galindo, Cáceres Luis J. Coordinador. 1998. Técnicas de Investigación en sociedad, cultura y comunicación. México: Editorial Pearson.

Mcconaughy, Stepahanie H. 2000. Life History Reports of Young Adults Previously Referred for Mental Health Services. Summarize of 22 paginate. Consultado 12/09/2001 en http://www.findarticles.com

Mejía, Arauz Rebeca., Sandoval, Sergio A. 1999. Tras Las Vetas De La Investigación Cualitativa, perspectivas y acercamientos desde la practica. México: ITESO.
Miles, Matthew. B., Huberman, A. Michael. 1994. Qualitative data analysis (2nd edition) Sage (Excerpts from pp. 2-11) London New Delhi.

Munduate, Lourdes., Ganaza, Juan., Peiro, Jose M., Euwema, Martín. 1999. Patterns of styles in conflict management and effectiveness. International Journal of conflict Management, v10n1, Page: 5-24. Consulted 12/09/2000 in Powered by Dialog.

Pillai, Rajnanadini. 1999. Fairness Perceptions and Trust as Mediators for Transformational and Transactional Leadership: A Twosample Study. Summarize of 38 paginate. Consultado el 02/15/2000 en http://www.findarticles.com

Rodríguez Valencia, Joaquín. 1999. Cómo administrar pequeñas y medianas empresas, México: Editorial ECAFSA.

Rojas, Soriano Raúl. 2000. Guía para Realizar Investigaciones Sociales., México: Editorial Plaza y Valdez.

Solís, Pérez Pedro C. 1998. Tesis de Doctorado: Modernización de las universidades públicas mexicanas: un estudio organizacional. México: UAM Iztapalapa.

Tamayo y Tamayo, Mario. 2001. El Proceso de la Investigación Científica. México: Editorial LIMUSA, S.A. de C.V.,

Taylor S. J. / Bogdan R. 1987. Introducción a los métodos cualitativos de investigación. Barcelona: Editorial Piados.

María del Socorro Borboa-Quintero, Doctora en Estudios Organizacionales por la Universidad Autónoma 
EXPERIENCIA EN METODOLOGÍA CUALITATIVA: HISTORIA DE VIDA

Metropolitana, Profesora e

Investigadora Titular "C" de

la Facultad de Contaduría y administración de la Universidad

Autónoma de Sinaloa, Miembro del

Sistema Nacional de Investigadores nivel I, y del Cuerpo Académico

Consolidado "Administración de Negocios y Desarrollo Regional”. 